\title{
Erratum to: RhoB is associated with the anti-angiogenic effects of celiac patient transglutaminase 2-targeted autoantibodies
}

\author{
Stefania Martucciello • Miha Lavric • Boglarka Toth • \\ Ilma Korponay-Szabo • Cristina Nadalutti • \\ Essi Myrsky • Tiina Rauhavirta • Carla Esposito • \\ Ana-Marija Sulic • Daniele Sblattero • \\ Roberto Marzari • Markku Mäki • Katri Kaukinen • \\ Katri Lindfors • Sergio Caja
}

Published online: 18 February 2012

(C) Springer-Verlag 2012

\section{Erratum to: J Mol Med}

$$
\text { DOI 10.1007/s00109-011-0853-0 }
$$

There is a mistake in the original publication in one of the co-authors names: Toth Boglarka. The correct name should be: Boglarka Toth (Givenname is Boglarka and Surname is Toth).
The online version of the original article can be found at http://dx.doi. org/10.1007/s00109-011-0853-0.

S. Martucciello - I. Korponay-Szabo • C. Nadalutti • E. Myrsky •

T. Rauhavirta $\cdot$ A.-M. Sulic $\cdot$ M. Mäki $\cdot$ K. Lindfors $(\bowtie) \cdot$ S. Caja

Pediatric Research Center, School of Medicine,

University of Tampere and Tampere University Hospital,

Finn-Medi 3, 33014, Tampere, Finland

e-mail: katri.lindfors@uta.fi

\section{S. Martucciello $\cdot$ C. Esposito}

Department of Chemistry, University of Salerno,

Fisciano, Italy

M. Lavric $\cdot$ R. Marzari

Department of Life Sciences, University of Trieste,

Trieste, Italy

\section{B. Toth}

Department of Biochemistry and Molecular Biology,

Medical and Health Science Center, University of Debrecen,

Debrecen, Hungary
I. Korponay-Szabo

Heim Pal Children's Hospital, Budapest and Department of

Pediatrics, Medical and Health Science Centre,

University of Debrecen,

Debrecen, Hungary

D. Sblattero

Department Medical Sciences and IRCAD,

University of Eastern Piedmont,

Novara, Italy

K. Kaukinen

School of Medicine, University of Tampere,

Finn-Medi 3, 33014, Tampere, Finland

\section{K. Kaukinen}

Department of Gastroenterology and Alimentary Tract Surgery, Tampere University Hospital,

Tampere, Finland 